\title{
Imagined Risk of Suffocation as a Trigger for Hyperventilation
}

\author{
Ilse Van Diest, PhD, Steven De Peuter, Ma, Stephan Devriese, PhD, Elke Wellens, Ma,
} Karel P. Van de Woestijne, MD, and Omer Van den Bergh, PhD

\begin{abstract}
Objective: Although hyperventilation has been hypothesized to play a role in many pathologies, its critical triggers remain poorly understood. The present experiment aimed to test whether stronger hyperventilation responses occur in response to suggested risk of suffocation compared with other fearful situations in high- and low-trait anxious women. Methods: Fractional end-tidal $\mathrm{CO}_{2}$-concentration $\left(\mathrm{FetCO}_{2}\right)$, respiratory frequency, and inspiratory volume were measured nonintrusively in high- $(n=24)$ and low- $(n=24)$ trait anxious women during imagery of 3 fear, 1 tension, 1 depressive, and 3 relaxation scripts. The fear scripts were equal in ratings of unpleasantness and arousal but differed regarding the inclusion of suggested risk of suffocation and entrapment. After each imagery trial, participants rated the emotional dimensions of pleasantness, arousal, and dominance and the vividness of their imagery. Results: Decreases in $\mathrm{FetCO}_{2}$ occurred in all fear scripts. High-trait anxious women showed a stronger reduction in $\mathrm{FetCO}_{2}$ compared with low-trait anxious women during the fear script suggesting risk of suffocation but not during the other fear scripts. This effect was unrelated to any of the self-reported fear ratings. Self-reported fear of entrapment was associated with an overall lower $\mathrm{FetCO}_{2}$ but not with enhanced reactivity to imagined entrapment. Conclusion: High-trait anxiety is associated with stronger respiratory responsivity to imagined risk of suffocation and may constitute a specific vulnerability factor for the development of panic disorder and claustrophobia. Key words: hyperventilation, trait anxiety, suffocation, fear, entrapment.
\end{abstract}

$\mathbf{F e t C O}_{2}=$ fractional concentration of end-tidal carbon dioxide; $\mathbf{f}_{\mathbf{r}}=$ respiratory frequency; $\mathbf{V i}=$ inspiratory volume; $\mathbf{C P S}=$ checklist for psychosomatic symptoms; STAI-T = trait version of the state-trait anxiety inventory; $\mathbf{F S}=$ fear of suffocation; $\mathbf{F R}=$ fear of restriction.

\section{INTRODUCTION}

$\mathrm{H}$ yperventilation is a breathing pattern that exceeds metabolic requirements (1): more $\mathrm{CO}_{2}$ is being expired than produced by the body, leading to a decreased $\mathrm{CO}_{2}$-pressure in the blood, and to a lower fractional end-tidal $\mathrm{CO}_{2}$ concentration $\left(\mathrm{FetCO}_{2}\right)$. Although hyperventilation has been hypothesized to play a role in many pathologies (panic disorder, chronic fatigue syndrome, multiple chemical sensitivity, chronic pain, somatization; 2-7) and can be considered an important cause for stress-related bodily complaints in general (3), its critical triggers remain poorly understood. Previous research from our group has shown that decreases in $\mathrm{FetCO}_{2}$ can be triggered during imagery of both pleasant and unpleasant high-arousal emotional situations, but not when imagining low arousal scenes $(8,9)$. This is in line with a psychophysiological perspective that respiratory reactivity reflects the general action tendency underlying an emotion, independently from specific emotional contents (10-12). In other words, an increase in ventilation during emotional states may be part of the increased arousal associated with emotions preparing the organism for action, irrespective of the direction of that action tendency (approach versus avoidance/escape).

However, given the obvious survival value of a satisfactory air supply, it would be adaptive for an organism to be especially reactive to (internal/external) cues signaling potential suffocation. Several observations are consistent with the existence of a primitive alarm system responding with intense

From the Department of Psychology, University of Leuven, Leuven, Belgium (I.V.D., S.D.P., S.D., E.W., O.V.d.B.); Faculty of Medicine, University of Leuven, Leuven, Belgium (P.V.d.W.).

Address correspondence and reprint requests to Ilse Van Diest, PhD, University of Florida, CSEA, 2800 SW. Archer Rd., Surge Bldg. 772, Gainesville, FL 32608. E-mail: Ilse.Vandiest@psy.kuleuven.ac.be

Ilse Van Diest is a postdoctoral researcher of the Fund of Scientific Research, Flanders, Belgium.

Received for publication October 13, 2004; revision received April 19, 2005.

DOI: 10.1097/01.psy.0000181275.78903.64 fear and hyperventilation to suffocation cues (13-26). If hyperventilation is part of an unconditioned fear response to suffocation, situations referring to risk of suffocation should trigger hyperventilation more easily than other fearful situations. As far as we know, this simple prediction has never been tested directly. Therefore, the present study aimed to compare responses in respiration and $\mathrm{FetCO}_{2}$ during imagery of fearful scripts with and without suffocation cues.

Whereas we previously showed (27) that there was no overall association between trait anxiety and end-tidal $\mathrm{CO}_{2}$ after careful control of confounding variables, we did find indications of stronger respiratory reactivity to mental load challenges in high-trait anxious persons. Interestingly, trait anxiety has also been found to be an important vulnerability factor for panic disorder (28). In addition, because there is some evidence that inter-individual differences in reactivity to suffocation stimuli are related to stress-induced modifications in brain functioning $(16,17)$, it would be interesting to see if particularly high-trait anxious participants show elevated reactivity when confronted with suffocation cues. Therefore, the present study also focused on the vulnerability for hyperventilatory responses of high-anxious compared with low-anxious participants.

$\mathrm{FetCO}_{2}$ and respiration were measured in 48 women during imagery of 8 different scripts. Three fear scripts were critical to address our principal research question: 1 suffocation and 2 control fear scripts. We hypothesized that stronger decreases in $\mathrm{FetCO}_{2}$ would occur for the fear script containing suffocation cues compared with the other fear scripts and that these effects would be more pronounced in high- as opposed to low-trait anxious participants.

In order to investigate whether, similarly to previous findings $(8,9)$, decreases in $\mathrm{FetCO}_{2}$ would occur during imagery of high- but not low-arousal scripts, 4 low-arousal scripts were included as well: 1 depressive and 3 relaxation scripts. A tense-anxiety script was additionally used to explore respiratory behavior under a more attentive defensive set associated with lower action tendencies (29).

\section{METHODS}

Methods were similar to previous studies from our group $(8,9)$. Therefore, a shortened description will be presented in this paper. 


\section{Participants}

Forty-eight women, all undergraduate students (17-19 years old), participated in return for course credit. They were selected from a larger pool on the basis of their scores on trait anxiety (see Procedure). The low-trait anxious group (24 women) consisted of students whose (raw) scores were in the lower quartile of the trait version of the state-trait anxiety inventory (STAI-T) $(<26)$, whereas the high-trait anxious participants $(24$ women) scored in the upper quartile of the STAI-T $(>44)$. Because too few men were available to participate, and because men and women differ in lung-volume parameters $(30,31)$, resulting in more error variance without permitting a balanced test for the effect of gender, men were not tested.

\section{Materials Scripts}

Eight scripts were presented to the participants. Three fear scripts (suffocation, entrapment-only, examination) were unpleasant and had strong action tendencies. A typical claustrophobic emotional imagery script was selected to investigate whether the risk of suffocation implied by a closed-in place with a limited supply of fresh air would trigger stronger respiratory responses than other control fear scripts. However, besides risk of suffocation, claustrophobic situations also typically contain elements of entrapment. Together with fear of suffocation (FS), fear of entrapment is considered an independent specific fear underlying claustrophobia $(18,32,33)$. Therefore, a second fear script included elements of entrapment, without the risk of suffocation associated with the "closed-in feeling" of a claustrophobic situation. The third fear script described fear of an examination and did not contain elements referring to risk of suffocation or of entrapment.

The "tension" script described someone participating in a television quiz show and aimed to trigger a tense effect with a weaker action tendency than the fear scripts. The depressive script was unpleasant and low in arousal, whereas the 3 relaxation scripts were neutral-positive and low in arousal. All scripts, with the exception of the entrapment-only and the tension scripts, have been used in a previous study (8). Because the inclusion of response information enhances the quality of the imagery $(9,34)$, all scripts contained suggestions of autonomic responses. In order not to interfere with spontaneous breathing patterns, suggestions referring directly to breathing responses that could be voluntarily controlled (eg, faster breathing) were avoided (see appendix).

\section{Apparatus and Physiological Recordings}

Participants were seated in a comfortable chair. Imagery scripts were presented via headphones plugged into the sound card of a PC.

FetCO $\mathrm{O}_{2}$ was measured using a nasal $\mathrm{CO}_{2}$-sampling cannula connected to a nondispersive infrared $\mathrm{CO}_{2}$-monitor with a resolution of $1 \mathrm{~mm} \mathrm{Hg}$ and an accuracy of $\pm 2 \mathrm{~mm} \mathrm{Hg}$ (Capnograph, Poet RC, Criticare). Ventilation was measured using a respiratory inductive plethysmograph (Respitrace Corporation Model 150, NY). The coils of the Respitrace were stuck to the skin, one around the rib cage under the axilla and the other on the abdomen above the pelvis. The calibration procedure consisted of a 5-minute period of breathing during which respiration was measured simultaneously by means of a pneumotachograph (Fleisch No 2, Switzerland) and the Respitrace. Multiple linear regression was performed over the calibration period, yielding the regression coefficients to be used for reconstruction of the volume out of the Respitrace signals (35). The multiple linear regression approach produces accurate volume data when $R^{2}>85 \%(31,36)$. A Labmaster Card and a PC processed the Respitrace signals and the $\mathrm{FetCO}_{2}$ signals with a sampling rate of $20 \mathrm{~Hz}$. The $\mathrm{FetCO}_{2}$ signal and the reconstructed volume were plotted as a function of time and visually inspected for technical abnormalities and movement artifacts.

Self-developed software extracted breathing pauses and irregularities and determined the following parameters for each breath: inspiratory (Ti) and expiratory time (Te) in seconds, inspiratory (Vi) and expiratory volume (Ve) in milliliters, and $\mathrm{FetCO}_{2}$ in percent. Respiratory frequency $\left(\mathrm{f}_{\mathrm{r}}\right)$ was calculated from the mean values of Ti, Te, and breathing pauses: $f_{r}=60 /(T i+T e+$ pauses).

\section{Self-Report Measures}

In a collective group session preceding the experiment, 439 undergraduates (351 women) had completed the STAI-T (zelfbeoordelingsvragenlijst; 37,38) and the Checklist for Psychosomatic Symptoms (CPS; 39). The CPS was used for exploratory reasons; it consists of 35 hyperventilation-related symptoms belonging to one of the following subsets: paresthesia, cerebral, cardiac, gastrointestinal, respiratory, anxiety, or unclassified symptoms. Four sensations that are rarely associated with hyperventilation, called "dummy symptoms," were added to this list: nasal congestion, joint pain, lower back pain, and aching eyes. Participants had to rate the extent to which they had experienced each symptom during the past year (never, rarely, regularly, often, very often).

During the experiment, participants rated the images evoked by the scripts on the basic affective dimensions of pleasantness (or valence), arousal, and dominance $(40,41)$, using the paper-and-pencil version of the 9-point SelfAssessment Manikin (42). Vividness of imagery was assessed by means of 9-point Likert-scales, ranging from "very" to "not vivid."

FS and fear of restriction (FR) were assessed using a translation of Rachman and Taylor's (33) suffocation and restriction scales. Each scale consists of 6 items with high loading on only 1 of both factors. Participants were asked to indicate the degree to which they would feel anxious in each of the situations. Responses were made on a 5-point scale, ranging from "not anxious" to "extremely anxious."

\section{Procedure}

The selected students were invited to participate in an experiment investigating physiological changes during imagery. On arrival, the experimenter told the participant that there were 8 imagery trials, each consisting of the same consecutive phases. These were explained as Baseline: When the light in the participant's room was dimmed, participants were to close their eyes, and 1 minute of relaxing music was presented through the headphones (a fragment of Sarabande, Goldberg Suite, E. Grieg). Participants were instructed simply to listen to the music. Script: During the following minute, the script was presented, and participants were asked to start the imagery as soon as they heard the text. Silence: The listening period was followed by $90 \mathrm{~s}$ of silence, during which they had to keep on imagining the script as vividly as possible. After $60 \mathrm{~s}$, a low level short auditory signal, announced during the instructions, was given as a reminder to continue the imagery as vividly as possible. Recovery: Thereafter followed a recovery period of $60 \mathrm{~s}$ with another piece of relaxing music (Gymnopédie no. 1, E. Satie). Participants were instructed to stop the imagery as soon as this music started, to keep their eyes closed, and to listen to the music. The light being turned on announced the end of an imagery trial.

Participants were told that physiological recordings would be made during the imagery and the purpose of the equipment was described. Participants were informed that the nose cannula sampled their expired air and sent it to an analyzer and that 2 coils would be attached to their rib cage and abdomen to detect changes in posture and muscle tonus of the trunk. The latter (misleading) explanation was given to avoid drawing the participant's attention to her respiration. After an informed consent was obtained, the Respitrace coils and the nose cannula were attached. The participant was asked to sit comfortably and was asked to maintain the same position during the entire experiment. Next, the calibration procedure was started, during which time participants wore a nose clip and breathed for 5 minutes through a mouthpiece that was connected to a pneumotachograph.

After the calibration phase, the importance of imagining the scene as vividly as possible was emphasized. Lang and colleagues (43) have demonstrated that prior training in so-called response orientation may enhance emotional imagery. Response orientation implies not only creating a detailed visual picture of the scene but trying to feel and experience the scene as if one were a real, active participant in it. After the first imagery trial, the experimenter asked whether she succeeded in imaging the scene vividly. Postimagery reports involving descriptions of one's own responses toward the situation (eg, feelings, bodily responses) were reinforced by the experimenter. Next, the participant was asked to fill out the pleasantness, arousal, dominance, and vividness ratings. Afterward, the experimenter explained that the 
other 6 remaining scripts would be presented according to the same scheme without any further interruption by the experimenter.

The presentation order of the scripts was semirandomized. In order to avoid repetition and boredom in the participants, high-arousal scripts were alternated with low-arousal scripts. The relax 1 script was always presented as the first script. The remaining low arousal scripts (relax 2, relax 3, depressive) were randomized across the third, fifth, and seventh positions, yielding 6 possible orders for low-arousal scripts. The fear and tension scripts were always presented on the second, fourth, sixth, and eight positions in a fixed sequence (suffocation, tension, entrapment-only, examination). However, the script with which this sequence started was randomized, yielding 4 possible orders of fear and tension scripts. One low and 1 high-trait anxious participant were randomly allocated to each of the 24 presentation orders that resulted from combining the 6 possible orders of the low-arousal scripts with the 4 orders of high-arousal scripts.

Finally, after the experiment the experimenter asked whether the participant had voluntarily controlled their breathing during 1 or more imagery trials and also whether they avoid elevators in real life because they feel anxious or uncomfortable in them. Answers to the latter question were coded as 0 ("never"), 1 ("sometimes"), or 2 (“always").

The scores on the FR and FS scales were obtained after the experiment. All 48 participants were invited by mail to complete the FS and FR scales. Forty-three participants returned a completed questionnaire to the experimenter.

\section{Data Reduction and Analysis}

In order to avoid potential confounding by presentation order or amount of response training, data of the first imagery trial (first relaxation script) were not analyzed.

\section{Manipulation Checks}

Omnibus ANOVAs on the self-reports (pleasantness, arousal, dominance, vividness) with Group (2 levels) as a between-subject and Script (7 levels) as a within-subject variable were run to check whether the scripts evoked the targeted emotional states and to ensure that they did not differ in vividness. Greenhouse-Geisser corrections were applied to these and all subsequent ANOVAs, including the Script factor (7 levels); unadjusted degrees of freedom, adjusted $p$ values, and $\varepsilon$ will be reported in the Result section.

We also checked also for baseline differences in breathing behavior: means of $\mathrm{FetCO}_{2}, \mathrm{f}_{\mathrm{r}}$, and Vi during baseline were analyzed in a similar design with Group (high/low trait anxiety) as a between-factor and Script (7 levels) as a within-subject factor.

\section{Planned Comparisons}

For the same breathing parameters, difference scores subtracting the baseline from the imagery period were calculated. The first $30 \mathrm{~s}$ of script presentation were not included in the imagery period. Specific hypotheses were addressed using planned contrasts. Testing planned comparisons has been recommended by several authors because of a better power and better articulation compared with an omnibus ANOVA (44-46). In addition, a significant omnibus effect is not required, provided that family-wise error is controlled for (45). This approach has an advantage in the present study design because the 3 critical fear scripts (suffocation, entrapment-only, examination) were embedded in a broader pool of imagery scripts that were not necessarily relevant to the particular question regarding risk of suffocation. Such cases have a clear advantage by allowing a better focus. Five planned contrasts were tested. In order to control for family-wise error, $\alpha$ was set at 0.01 (Bonferonni correction).

Four contrasts addressed our hypotheses concerning risk of suffocation as a specific trigger for hyperventilation. The suffocation script was contrasted with each of the other fear scripts (entrapment-only, examination), and both contrasts were tested within each of both anxiety groups. A fifth, additional contrast tested whether high-arousal scripts (examination, suffocation, entrapment-only, tension) led to a reduction in $\mathrm{FetCO}_{2}$ compared with low-arousal scripts (depression, relax 2, relax 3).

\section{Exploratory Analyses}

In a set of exploratory analyses, correlations between trait anxiety, FS, FR, and the CPS on the one hand and baseline $\mathrm{FetCO}_{2}$ and change score in $\mathrm{FetCO}_{2}$ during imagery of the fear scripts on the other hand were calculated. Only significant effects will be reported.

\section{RESULTS \\ Manipulation Checks}

The arousal and pleasantness ratings confirmed that the scripts generally evoked the targeted emotional states (see Table 1). The fear and tension scripts were rated on the negative side of the pleasantness scale and high in arousal. The depressive script was rated negatively but low in arousal. The relaxation scripts were rated positively and low in arousal. Main effects of Script were significant for all ratings $(F(6,276)$ were for pleasantness: 293, $p<.001, \varepsilon=0.80$; for arousal $146, p<.001, \varepsilon=.80$; for dominance: $71, p<.001, \varepsilon=$ 0.63 ; for vividness: $7.10, p<.001, \varepsilon=0.89)$. A significant Group $\times$ Script interaction effect $(F(6,276)=3.26, p<.05$, $\varepsilon=0.63$ ) emerged only for the dominance ratings. Follow-up comparisons showed that the high-anxious group tended to feel less dominant during the unpleasant scripts (depressive, examination, suffocation, entrapment-only, tension) than the

TABLE 1. Mean Ratings for Each Imagery Trial $(N=48)$

\begin{tabular}{|c|c|c|c|c|c|c|c|}
\hline & $\mathrm{D}$ & Ex & Suf & Entr & Relax 2 & Relax 3 & Tens \\
\hline \multicolumn{8}{|c|}{ Pleasantness } \\
\hline $\mathrm{M}$ & 7.35 & 7.46 & 7.83 & 7.67 & 1.65 & 1.63 & 5.85 \\
\hline SD & 1.39 & 1.34 & 1.19 & 1.26 & 0.98 & 1.12 & 1.62 \\
\hline \multicolumn{8}{|c|}{ Arousal } \\
\hline $\mathrm{M}$ & 2.67 & 6.08 & 6.40 & 5.94 & 1.40 & 1.44 & 5.65 \\
\hline SD & 1.62 & 1.54 & 1.71 & 1.60 & 0.96 & 1.05 & 1.91 \\
\hline \multicolumn{8}{|c|}{ Dominance } \\
\hline M & 4.50 & 3.63 & 2.50 & 2.92 & 7.71 & 7.90 & 4.35 \\
\hline SD & 2.53 & 2.00 & 1.54 & 1.83 & 1.76 & 1.79 & 2.01 \\
\hline \multicolumn{8}{|c|}{ Vividness } \\
\hline $\mathrm{M}$ & 6.54 & 6.73 & 6.67 & 6.81 & 7.52 & 7.52 & 5.77 \\
\hline SD & 2.12 & 1.67 & 1.86 & 1.57 & 1.43 & 1.77 & 1.84 \\
\hline
\end{tabular}

$\mathrm{D}=$ depressive script; Ex = examination script; Suf = suffocation script; Entr = entrapment-only script; Relax 2 = sea script; Relax $3=$ living room script; Tens $=$ tension script. Lower values indicate less aroused, less pleasant, less dominant, and less vivid imagery, all on a 1-to-9 scale. 
TABLE 2. Baseline Values of $\mathrm{FetCO}_{2}$ (in \%), Respiratory Frequency (per Minute) and Inspiratory Volume (in ml) and Respective Change Scores $(\Delta)$ From Preimagery Baseline to Imagery $(N=48)$

\begin{tabular}{|c|c|c|c|c|c|c|c|}
\hline & D & Ex & Suf & Entr & Relax 2 & Relax 3 & Tens \\
\hline \multicolumn{8}{|c|}{ FetCO } \\
\hline$M$ & 5.11 & 5.10 & 5.13 & 5.11 & 5.09 & 5.10 & 5.08 \\
\hline SD & 0.49 & 0.46 & 0.47 & 0.47 & 0.46 & 0.46 & 0.50 \\
\hline \multicolumn{8}{|l|}{$f_{r}$} \\
\hline$M$ & 15.81 & 15.71 & 15.54 & 15.69 & 15.29 & 15.70 & 15.66 \\
\hline SD & 2.85 & 2.68 & 2.86 & 3.01 & 2.70 & 2.74 & 2.76 \\
\hline \multicolumn{8}{|l|}{$\mathrm{Vi}$} \\
\hline M & 341 & 338 & 354 & 344 & 356 & 350 & 350 \\
\hline SD & 82 & 73 & 95 & 71 & 83 & 85 & 96 \\
\hline \multicolumn{8}{|c|}{$\Delta \mathrm{FetCO}_{2}$} \\
\hline M & 0.05 & -0.14 & -0.27 & -0.23 & 0.10 & 0.09 & -0.03 \\
\hline SD & 0.15 & 0.27 & 0.45 & 0.41 & 0.19 & 0.11 & 0.28 \\
\hline \multicolumn{8}{|l|}{$\Delta \mathrm{f}_{\mathrm{r}}$} \\
\hline$M$ & 0.32 & 2.24 & 3.87 & 3.45 & -0.12 & -0.24 & 1.76 \\
\hline SD & 2.61 & 4.24 & 6.60 & 5.58 & 2.39 & 1.86 & 3.71 \\
\hline \multicolumn{8}{|l|}{$\Delta \mathrm{Vi}$} \\
\hline M & -6.58 & 5.21 & -11.78 & -1.01 & -10.61 & -6.20 & -14.69 \\
\hline SD & 64.01 & 72.63 & 92.59 & 80.98 & 57.36 & 52.89 & 75.90 \\
\hline
\end{tabular}

$\mathrm{D}=$ depressive script; Ex = examination script; Suf = suffocation script; Entr = entrapment-only script; Relax 2 = sea script; Relax $3=$ living room script; Tens $=$ tension script; FetCO $\mathrm{O}_{2}=$ fractional end-tidal $\mathrm{CO}_{2}$ concentration; $\mathrm{f}_{\mathrm{r}}=$ frequency of respiration; $\mathrm{Vi}=$ inspiratory volume .

low anxious group, $F(1,46)=6.31, p<.025$, but no difference among both anxiety groups appeared for the pleasant $($ relax 2, relax 3$)$ scripts $(F(1,46)=1.35$, n.s. $)$.

There were no baseline differences among the seven imagery trials for $\mathrm{FetCO}_{2}, \mathrm{f}_{\mathrm{r}}$, or Vi (see Table 2 for means and standard deviations), nor were there any significant effects involving Group.

Table 2 also displays the mean change scores in $\mathrm{FetCO}_{2}, \mathrm{f}_{\mathrm{r}}$, and $\mathrm{Vi}$, and standard deviations during imagery. The changes in $\mathrm{FetCO}_{2}$ in the tension, the depressive, and both relaxation scripts were only minor and did not surpass the values of normal intraindividual variability in $\mathrm{FetCO}_{2}$ during rest (48). Relevant decreases in FetCO $\mathrm{C}_{2}$ and increases in $\mathrm{f}_{\mathrm{r}}$ occurred in the 3 fear scripts.

\section{Planned Comparisons}

\section{Suffocation Versus Other Fear Scripts}

For the high-trait anxious group, stronger decreases in FetCO $\mathrm{C}_{2}$ occurred in the suffocation script compared with the other fear scripts. This was not found for the lowanxious participants (see Figure 1 and Table 3). Similarly, the suffocation script triggered stronger increases in $f_{r}$ than the examination script in high, but not in low-trait anxious participants (Table 3). Changes in $\mathrm{f}_{\mathrm{r}}$ did not differ between the suffocation and the entrapment-only scripts for either group. No significant effects on Vi responses during imagery were found.

Self-reported pleasantness and arousal ratings for the suffocation script did not differ from ratings for the other fear scripts in either of the anxiety groups (see Table 3 for statistics). The suffocation script was rated lower in dominance than the examination script, and this was the case for both anxiety groups (see Table 3 ).

\section{High- Versus Low-Arousal Scripts}

The high-arousal scripts (suffocation, entrapment-only, examination, tension) were associated with a greater reduction in FetCO ${ }_{2}$ and a higher $\mathrm{f}_{\mathrm{r}}$ than the low-arousal scripts (depression, relax 2, relax 3 ). The former were also rated higher in arousal, more unpleasant, and lower in dominance than the latter (see Table 1 for means and standard deviations and Table 3 for statistics).

\section{Correlational Analyses}

Participants scoring high on FR had lower absolute baseline levels of $\mathrm{FetCO}_{2}(r=-0.34, N=43, p<.05)$. Change scores from baseline to imagery in $\mathrm{FetCO}_{2}$ during imagery of the suffocation, entrapment-only, or examination script were not related to FR or to FS. A stronger decrease in $\mathrm{FetCO}_{2}$ during imagery of the suffocation script was associated with

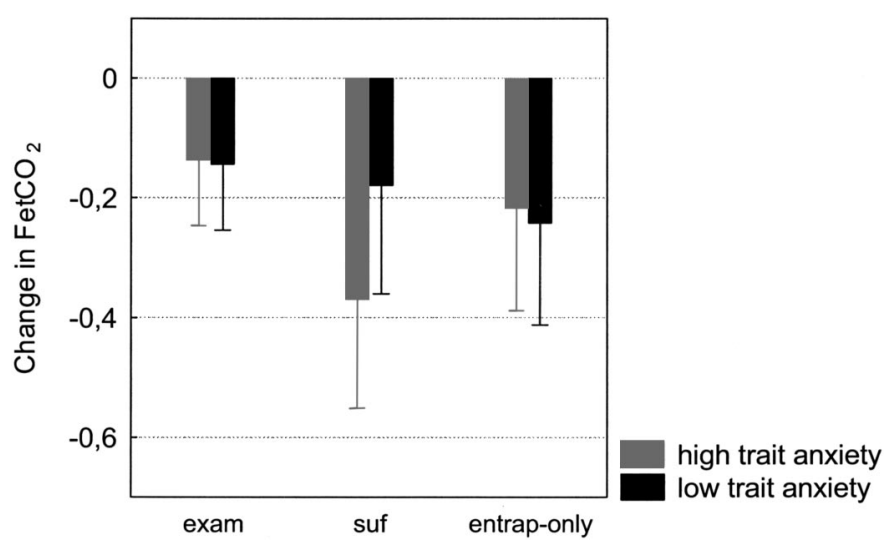

Figure 1. Mean change in $\mathrm{FetCO}_{2}$ in high- and low-trait anxious participants during imagery of the examination, the suffocation (suf), and the entrapmentonly (entrap-only) scripts. Vertical bars denote 0.95 confidence intervals. 
TABLE 3. Within Subject $t$ 's for the Planned Comparisons

\begin{tabular}{|c|c|c|c|c|c|c|c|}
\hline \multirow{2}{*}{ Contrast } & \multirow{2}{*}{$d f$} & \multicolumn{6}{|c|}{$t$} \\
\hline & & $\mathrm{FetCO}_{2}$ & $f_{r}$ & Vi & Val & Arous & Dom \\
\hline 1. Suf-Entr, High anxious group & 23 & $2.9^{a}$ & 1.6 & 0.3 & 0.6 & 0.1 & -1.3 \\
\hline 2. Suf-Entr, Low anxious group & 23 & -1.2 & -0.4 & -1.6 & 0.6 & 2.4 & -1.6 \\
\hline 3. Suf-Ex, High anxious group & 23 & $3.3^{a}$ & $3.2^{a}$ & 0.11 & 1.2 & 0.3 & $-2.7^{a}$ \\
\hline 4. Suf-Ex, Low anxious group & 23 & 0.50 & 0.22 & 2.1 & 1.5 & 1.3 & $-3.6^{a}$ \\
\hline 5. (D, Relax 1, 2, 3)-(Suf, Entr, Ex, Tens) & 47 & $6.3^{a}$ & $-4.2^{a}$ & -0.2 & $-27.3^{a}$ & $22.6^{a}$ & $11.0^{a}$ \\
\hline
\end{tabular}

$\mathrm{D}=$ depressive script; Ex = examination script; Suf = suffocation script; Entr = entrapment-only script; Relax $2=$ sea script; Relax $3=$ living room script; Tens $=$ tension script; Val $=$ valence; Arous $=$ Arousal; Dom $=$ Dominance; FetCO $\mathrm{CO}_{2}=$ fractional end-tidal $\mathrm{CO}_{2}$ concentration; $\mathrm{f}_{\mathrm{r}}=$ frequency of respiration; $\mathrm{Vi}=$ inspiratory volume.

${ }^{a} p<.01$.

more respiratory $(r=-0.32, N=48, p<.05)$ and paresthesia $(r=-0.34, N=48, p<.05)$ symptoms in daily life, but not to the other symptom groups of the CPS (anxiety, cardiac, gastrointestinal, cerebral, and unclassified symptoms) and was only marginally significantly $(r=-0.26, N=48$, $p<.08)$ related to trait anxiety.

\section{DISCUSSION}

\section{Suffocation Risk}

As hypothesized, specific comparisons between fear scripts showed that the reduction in $\mathrm{FetCO}_{2}$ was particularly pronounced in high- but not in low-trait anxious participants during imagery of the suffocation script. Although the observed changes in $\mathrm{FetCO}_{2}$ during imagery of the suffocation script remain small compared with clinical levels of hyperventilation, this pattern of results suggests that the interaction between dispositional anxiety and situational risk of suffocation may constitute a vulnerability factor for panic disorder and for claustrophobia. Interestingly, both disorders have been found to benefit from disconfirmation of misappraisals of bodily sensations in cognitive-behavioral therapy (48). Hyperventilation may be a common, important producer of bodily symptoms in both disorders. Exploratory correlational analyses further support that high $\mathrm{FetCO}_{2}$ responsiveness during imagery in the laboratory may be a valid predictor of responsiveness in real life: $\mathrm{FetCO}_{2}$ decreases during imagery of the suffocation script were specifically related to the report of typical hyperventilation (respiratory and paresthesia) symptoms in daily life.

The stronger $\mathrm{FetCO}_{2}$ reactivity in high-anxious participants during suffocation imagery was not paralleled by any of the self-report measures. Both anxiety groups had similar scores on the FS scale and a similar number of participants who were truly phobic of elevators. Respiratory responses were highly variable in the small sample of participants answering positively on the postexperimental question regarding avoidance behavior for elevators: the decrease in $\mathrm{FetCO}_{2}$ for phobic subjects ranged from $-0.18 \%$ to $-0.97 \%$ in the 5 high-trait anxious and from $-0.19 \%$ to $-1.31 \%$ in the 3 low-anxious participants. However, analyses excluding these claustrophobic participants did not yield another pattern of results. Furthermore, the enhanced respiratory responsiveness of the high-trait anx- ious group in the suffocation script was not related to more vivid imagery in that group or to corresponding effects found on the ratings of pleasantness, arousal, dominance, or vividness. A possible ceiling effect for the pleasantness ratings of the suffocation script may account for these latter findings. Indeed, the majority of the participants scored this script close to the negative end point of the scale. $\mathrm{FetCO}_{2}$ may be a more sensitive measure to differentiate between extreme fear levels. Alternatively, this finding may be an example of discordance between self-report and physiological measures. This pattern is quite common and has been suggested to be related to pathology (49).

The results on $\mathrm{FetCO}_{2}$ do not exactly parallel the $\mathrm{f}_{\mathrm{r}}$ results. As can be seen from Table 3, the suffocationorentrapmentonly contrast in the high-anxiety group was significant for FetCO $\mathrm{C}_{2}$ but not for $\mathrm{f}_{\mathrm{r}}$. This is likely a consequence of $\mathrm{FetCO}_{2}$ being a more sensitive measure, resulting from the combined and coordinated changes in several other breathing parameters. In other words, changes in $f_{r}$ and volume may not reach statistical significance by themselves, but their combined effect may influence $\mathrm{FetCO}_{2}$ in a highly significant way. In that respect, $\mathrm{FetCO}_{2}$ is a more important and relevant output measure, documenting whether the continuous and interdependent variations in the breathing pattern are metabolically appropriate.

Theoretically, it would have been interesting also to have included a script with risk of suffocation without entrapment, eg, a script of someone experiencing an asthma attack. However, such a script would have drawn too much attention toward respiratory sensations and behavior, which changes the breathing pattern by itself (see 31 ) or likely induces voluntarily control of breathing in order to mimic the attack. For the same reason, we opted to relate the risk of suffocation in the imagery script to external cues rather than to interceptive cues, although the latter might have been more relevant for panic disorder.

\section{Entrapment Only}

Trait-like FR was not associated with an enhanced respiratory reactivity to imagined entrapment. However, people with a higher FR had a consistently lower $\mathrm{FetCO}_{2}$ during the entire experiment. A likely explanation for this may be the 
actual movement restriction in the experimental context. Indeed, several cables connecting the participant's torso to the physiological recording systems and the experimenter's instructions not to move or change position (see procedure) may have triggered fear and an increased respiration in participants with FR. This finding may be important for nonambulatory psychophysiological research in general, in which participants, in addition to instructions not to move, are typically physically restricted by measurement devices such as electrodes. Because of the direct relationship between arterial $\mathrm{PCO}_{2}$ and cerebral blood flow (50), the finding may have particular relevance for brain-imaging studies.

Similar to previous findings $(8,9)$, we observed a decreased FetCO $\mathrm{O}_{2}$ and an increased $\mathrm{f}_{\mathrm{r}}$ during imagery of fearful scripts compared with low-arousal (depressive and relaxation) scripts. A tension script that aimed to trigger a tense-attentive defensive response set with a low action tendency was introduced. Although this script was also rated rather negatively and high on arousal (see Table 1), it triggered only a very weak response in $\mathrm{FetCO}_{2}$, which is in line with the idea that respiration generally covaries with the action tendency underlying an emotion.

In summary, our findings further corroborate the general idea that respiratory responses, and $\mathrm{FetCO}_{2}$ in particular, reflect the action tendency underlying an emotion $(8,10-12)$. Although high-trait anxious participants did not report being more fearful about situations with a possible risk of suffocation than low-trait anxious participants, only the former responded with stronger increases in respiration and concomitant decreases in $\mathrm{FetCO}_{2}$ to imagined risk of suffocation. Adding nonrespiratory physiological (eg, cardiovascular) measurements in future studies would be interesting to determine whether the enhanced responsiveness of high-trait participants to situational risk of suffocation is specific for the respiratory system.

\section{Appendix: Imagery Scripts Depression}

It's a gray morning and you wake up. You keep your eyes closed. You should get up, but you don't feel like starting the day. The thought of starting your daily routine makes you feel miserable. What's the use? Your body feels tired and heavy. You feel empty inside and apathetic. Your head feels dull and you can't think straight. What's more, you don't want to think. Actually, you wish it were evening again, so that you could go to bed again and not feel this miserable. You feel lonely. Nobody understands you.

\section{Examination}

You're studying. You look up from your desk and you see the huge pile of books on your bookcase. You must still study them all, and exams will start soon. You're getting warm. You're realizing you're running out of time. You feel nervous. All your best friends are better off. You start sweating and your heart is pounding strongly and rapidly. You're anxious and nervous. You cannot get the exams and that pile of books out of your mind. All this worrying makes studying even harder. Failing your exams would be a disaster. Your heart is pounding heavily and your chest is tight.

\section{Suffocation}

You are alone in an elevator. Suddenly, the elevator stops and the light goes off. You're caught in between 2 floors. The door remains closed. It's a little place without any ventilation. You're warm and you feel a bit tight in the chest. You want to get out as soon as possible. Anxious feelings come over you. You're sweating and you feel your heart pounding strongly. There is too little air in the elevator. In despair, you're pushing on all the buttons, but nothing happens. Your body is streaming with perspiration and your chest feels compressed. There is almost no air available anymore in this little place. You pull the door with all your strength. Your heart pounds wildly and you can feel it all over your body. The only thing you can think of is: "I want to get out."

\section{Entrapment Only}

It's early in the evening and you're walking alone along a path in the forest. A strong wind is blowing. All of a sudden, there is a squall and you hear a loud, cracking noise. Before you realize what is happening, you're down and a heavy branch lands on your right leg. Your leg is OK, but you find you're stuck under the branches. You start sweating. You're really stuck and will never get out without help. This idea makes you feel panicky. Your heart is pounding strongly and your chest feels compressed. You feel warm and tight. You want to get out, but that's impossible. Nobody really knows you're here, and it will take a long time before anyone will notice you're missing. Your heart is beating like crazy. You want to escape, but you're stuck.

\section{Relax 1}

You're sitting in the backseat of a moving car. The driver drives relaxed and is well concentrated on the road. The radio is playing softly and the passengers are not very talkative. You're staring through the window. It's a calm, misty day. There's little traffic on this long straight road in the country. The lampposts pass you by in a steady rhythm. From time to time you can see a cyclist or a pedestrian. The fog hangs above the fields and the meadows along the road. In the distance, you can see the vague contours of a forest.

\section{Relax 2}

You are in an apartment overlooking the sea. You just got back from a walk on the beach and you're relaxing in a chair. You took off your shoes and the radio is playing in the background. It's springtime. The sun shines inside through the window and gives your body comfortable warmth. Sitting back, you look out of the window watching the sea. The sea is calm. The tide is high but the beach still stretches out a good way. Some children are building a sandcastle. A group of people with a dog is passing by. They make the seagulls fly away from the sand.

\section{Relax 3}

Sunday afternoon at home. In your most relaxing chair, you're at ease reading a book. It's cozy inside the living room and a fragrant cup of coffee sits next to you. Sitting back, relaxed, you look out of the window. It's a sunny autumn day outside. Red and brown leaves drift slowly down from the trees. A passing car blows them up again. A little breeze takes them further away. A few walkers are passing by. You sip your coffee and put the cup on the table again. You pick up your book again.

\section{Tension}

You're participating in a television quiz show. You've already earned a huge amount of money. In a moment, the final test will start: a series of difficult mental arithmetic questions that you should answer correctly and rapidly. If you succeed, the amount of money will be doubled. If you fail, you will lose everything. You're tense, completely focused. The quiz master calls for silence. The music and hubbub fade; it is silent now. You're extremely focused. "Are you ready?" the quiz master is asking; "Here are the questions." You're awaiting the questions ... you hardly dare to move ... you feel paralyzed ....

The authors thank Dr. Carson Smith and Tammy Silakowski for his comments on this article. 


\section{REFERENCES}

1. Gardner WN. The pathophysiology of hyperventilation disorders. Chest 1996;109:516-34.

2. Ley R. Dyspneic-fear and catastrophic cognitions in hyperventilatory panic attacks. Behav Res Ther 1989;5:549-54.

3. Sharpe M, Bass C. Pathophysiological mechanisms in somatization. Int Rev Psychiatry 1992;4:81-97.

4. Bazelmans E, Bleijenberg G, Vercoulen JHHM, van der Meer JWM, Folgering H. The chronic fatigue syndrome and hyperventilation. J Psychosom Res 1997;43:371-7.

5. Papp LA, Klein DF, Gorman JM. Carbon dioxide hypersensitivity, hyperventilation and panic disorder. Am J Psychiatry 1993;150:1149-57.

6. Van den Bergh O, Stegen K, Van Diest I, Raes C, Strulens P, Eelen P, Veulemans H, Van de Woestijne KP, Nemery B. Acquisition and extinction of somatic symptoms in response to odours: a pavlovian paradigm relevant to multiple chemical sensitivity. Occup Env Med 1999;56: 295-301.

7. Wilhelm FH, Gevirtz R, Roth WT. Respiratory dysregulation in anxiety, functional cardiac, and pain disorders: assessment, phenomenology, and treatment. Behav Modif 2001;25:513-45.

8. Van Diest I, Winters W, Devriese S, Vercamst E, Han JN, Van de Woestijne KP, Van den Bergh O. Hyperventilation beyond fight/flight: respiratory responses during emotional imagery. Psychophysiology 2001; 38:961-8.

9. Van Diest I, Proot P, Van de Woestijne KP, Han JN, Devriese S, Winters W, Van den Bergh O. Critical conditions for hyperventilation responses: the role of autonomic response propositions during emotional imagery. Behav Modif 2001;25:621-39.

10. Boiten F, Frijda NH, Wientjes CJ. Emotions and respiratory patterns: review and critical analysis. Int J Psychophysiol 1994;17:103-28.

11. Dudley DL, Pitts-Poarch AR. Psychophysiologic aspects of respiratory control. Clin Chest Med 1980;1:131-43.

12. Nyklicëk I, Thayer JF, Van Doornen LJP. Cardiorespiratory differentiation of musically-induced emotions. J Psychophysiol 1997;11:304-21.

13. Hilton SM. The defense-arousal system and its relevance for circulatory and respiratory control. J Exp Biol 1982;100:159-74.

14. Klein DR. Testing the suffocation false alarm theory of panic disorder. Anxiety 1994;1:1-7.

15. Klein DR. False suffocation alarms, spontaneous panics, and related cognitions. Arch Gen Psychiatry 1993;50:306-17.

16. Battaglia M. Beyond the usual suspects: a cholinergic route for panic attacks. Mol Psychiatry 2002;7:239-46.

17. Battaglia M, Bertella S, Ogliari A, Bellodi L, Smeraldi E. Modulation by muscarinic antagonists of the response to carbon dioxide challenge in panic disorder. Arch Gen Psychiatry 2001;58:114-9.

18. Rachman RS. Claustrophobia. In: Davey GC, ed. Phobias: A Handbook of Theory, Research and Treatment. New York: Wiley; 1997:163-82.

19. Campbell D, Sanderson RE, Laverty SG. Characteristics of a conditioned response in human subjects during extinction trials following a single traumatic conditioning trial. J Abnorm Soc Psychol 1964;6:627-39.

20. Carr RE. Panic disorder and asthma. J Asthma 1999;36:143-52.

21. Smoller JW, Simon NM, Pollack MH, Kradin R, Stern T. Anxiety in patients with pulmonary disease: comorbidity and treatment. Sem Clin Neuropsychiatry 1999;4:84-97.

22. Biber B, Alkin T. Panic disorder subtypes: differential responses to $\mathrm{CO}_{2}$ challenge. Am J Psychiatry 1999;156:739-44.

23. Hegel MT, Ferguson RJ. Psychophysiological assessment of respiratory function in panic disorder: evidence for a hyperventilation subtype. Psychosom Med 1997;59:224-30.

24. Briggs AC, Stretch DD, Brandon S. Subtyping of panic disorder by symptom profile. Br J Psychiatry 1993;163:201-9.

25. Telch MJ, Jacquin K, Smits JAJ, Powers MB. Emotional responding to hyperventilation as a predictor of agoraphobia status among individuals suffering from panic disorder. J Behav Ther 2003;34:161-70.

26. Stegen K, De Bruyne K, Rasschaert W, Van de Woestijne KP, Van den
Bergh O. Fear-relevant images as conditioned stimuli for somatic complaints, respiratory behavior, and reduced end-tidal $\mathrm{pCO}_{2}$. J Abnorm Psychol 1999;108:143-52.

27. Van Diest I, Vuerstaek S, Corne I, De Peuter S, Devriese S, Van de Woestijne KP, Van den Bergh O. Resting end-tidal $\mathrm{CO}_{2}$ and negative affectivity. Psychosom Med 2003;65:976-83.

28. Hayward C, Killen J, Kraemer HC, Taylor CB. Predictors of panic attacks in adolescents. J Am Aced Child Adolesc Psychiatry 2000;39:207-14.

29. Lang PJ, Bradley MM, Cuthbert BN. Motivated attention: affect, activation and action. In: Lang PJ, Simons RF, Balaban M, eds. Attention and Orienting: Sensory and Motivational Processes. Mahwah, NJ: Lawrence Erlbaum Associates; 1997:97-136.

30. Dhokalia A, Parsons DJ, Anderson DE. Resting end-tidal $\mathrm{CO}_{2}$ association with age, gender, and personality. Psychosom Med 1998;60:33-7.

31. Han JN, Stegen K, Cauberghs M, Van de Woestijne KP. Influence of awareness of the recording of breathing on respiratory pattern in healthy humans. Eur Respir J 1997;10:161-6.

32. Harris LM, Robinson J, Menzies RG. Evidence for fear of restriction and fear of suffocation as components of claustrophobia. Behav Res Ther 1999;37:155-9.

33. Rachman S, Taylor S. Analyses of claustrophobia. J Anxiety Disord 1993;7:281-91.

34. Lang PJ. A bio-informational theory of emotional imagery. Psychophysiology $1979 ; 16: 495-512$.

35. Strömberg NO, Dahlbäck GO, Gustafsson PM. Evaluation of various models for respiratory inductance plethysmography. J Appl Physiol 1993; 74:1206-11.

36. Han JN. Breathing Patterns in Anxiety Disorders: Is There a Hyperventilation Syndrome? Leuven: Leuven University Press; 1995.

37. Van der Ploeg HM. Validity of the Zelf-Beoordelings-Vragenlijst (a Dutch version of the Spielberger State-Trait Anxiety Inventory). Nederlands Tijdschrift Voor Psychologie Haar Grensgebieden 1980;35:243-9.

38. Spielberger CD, Gorsuch RL, Lushene RE. Manual for the State-Trait Anxiety Inventory. Palo Alto: Consulting Psychologists Press; 1970.

39. Wientjes CJE, Grossman P. Overreactivity of the psyche or the soma? interindividual associations between psychosomatic symptoms, anxiety, heart rate, and end-tidal partial carbon dioxide pressure. Psychosom Med 1994;56:533-40.

40. Lang PJ, Bradley MM, Cuthbert BN. Emotion, attention, and the startle reflex. Psychol Rev 1990;97:377-95.

41. Mehrabian A, Russell JA. An Approach to Environmental Psychology. Cambridge, MA: MIT Press; 1974.

42. Bradley MM, Lang PJ. Measuring emotion: the Self-Assessment Manikin (SAM) and the semantic differential. J Behav Ther Exp Psychiatry 1994;25:49-59.

43. Lang PJ, Kozak MJ, Miller GA, Levin DN, McLean A. Emotional imagery: conceptual structure and pattern of somato-visceral response. Psychophysiology 1980;17:179-92.

44. Abelson P, Prentice DA. Contrast tests of interaction hypotheses. Psychol Methods 1997;2:315-28.

45. Myers JL, Well AD. Research Design and Statistical Analysis. Hillsdale, NJ: Erlbaum; 1995.

46. Rosenthal R, Rosnow RL. Contrast Analysis: Focused Comparisons in the Analysis of Variance. New York: Cambridge University Press; 1985.

47. Shea SA. Life without ventilatory chemosensitivity. Respir Physiol 1997; 110:199-210.

48. Craske MG, Mohlman J, Yi J, Glover D. Treatment of claustrophobia and snake/spider phobias: fear of arousal and fear of context. Behav Res Ther 1995;33:197-203.

49. Cuthbert BN, Lang PJ, Strauss C, Drobes D, Patrick CJ, Bradley MM The psychophysiology of anxiety disorder: fear memory imagery. Psychophysiology 2003;40:407-22.

50. Michenfelder JD. Anesthesia and the Brain: Clinical, Functional, Metabolic and Vascular Disorders. New York: Churchill Livingstone; 1988: 51-61. 